\title{
Efeito de Reforçadores Condicionados Específicos em Classes Ordinais em Humanos
}

\author{
Grauben José Alves de Assis ${ }^{1}$ \\ Núcleo de Teoria e Pesquisa do Comportamento da Universidade Federal do Pará, \\ Belém, PA, Brasil \\ Carla Motta Mendes \\ Universidade Federal do Pará, Belém, PA, Brasil \\ Paulo Sérgio Teixeira do Prado \\ Departamento de Psicologia da Educação da Universidade Estadual Paulista Júlio \\ de Mesquita Filho, Marília, SP, Brasil
}

\section{Resumo}

A explicação segundo a qual classes de equivalência são formadas por contingências de reforço, tem como implicação que respostas e estímulos reforçadores também seriam incluídos entre seus membros. Suporte empírico a essa tese vem de experimentos utilizando reforçadores específicos para cada classe potencial, tanto em contexto de discriminação condicional, quanto de discriminação simples, neste último caso em combinação com reversões sistemáticas, em que as funções $\mathrm{S}^{+}$e $\mathrm{S}^{-}$dos estímulos discriminativos são constantemente invertidas. Em ambos os casos, testes apropriados evidenciaram a inclusão dos estímulos reforçadores como membros das classes de equivalência. Esses resultados permitem supor que estímulos reforçadores também participariam de classes ordinais. Estas são definidas pelas propriedades: assimetria, irreflexividade, transitividade, substituibilidade e conectividade. De caráter preliminar, o presente estudo testou uma estratégia experimental como um primeiro passo para uma investigação sobre a inclusão de estímulos reforçadores como membros de classes ordinais. Seu objetivo foi investigar o efeito de reforçadores condicionados específicos na formação de classes ordinais. Na presença de um estímulo condicional, seis universitários deviam sequenciar formas não representacionais numa determinada ordem, ao que seguia-se a apresentação de um trecho musical. Na presença de outro estímulo condicional, os mesmos estímulos deviam ser sequenciados na ordem inversa, produzindo outro trecho musical. Testes evidenciaram que além da emergência das propriedades definidoras de classes ordinais, os trechos musicais intercambiaram sua função reforçadora aos estímulos condicionais. Sendo a estratégia experimental eficaz nesse sentido, o próximo passo será verificar sua eficácia para promover a inclusão de reforçadores como membros de classes sequenciais.

Palavras-chave: Classes ordinais, reforçadores condicionados específicos, humanos.

\section{Effect of Conditioned Reinforcers Specific in Ordinal Classes in Humans}

\begin{abstract}
The explanation according to which equivalence classes are formed by contingencies of reinforcement, has the implication that responses and reinforcer stimuli would also be included among its members.
\end{abstract}

Endereço para correspondência: Universidade Federal do Pará, Núcleo de Teoria e Pesquisa do Comportamento, Laboratório de Psicologia, Rua Augusto Correa, s/n, Guamá, Belém, PA, Brasil 66075-900. Fone: (91) 32018488; Fax: (91) 320-17662. E-mail: ggrauben@gmail.com e pradopst@gmail.com 
Empirical support for this theory comes from experiments using specific reinforcers to each potential class, both in the context of conditional discrimination as in the simple discrimination context, in the latter case in combination with systematic reversals, in which $\mathrm{S}+$ and $\mathrm{S}$ - functions of the discriminative stimuli are constantly reversed. In the two cases, appropriated tests showed the inclusion of reinforcer stimuli as members of the equivalence classes. These results encourage hypothesize that reinforcer stimuli also participate in ordinal classes, which are defined by the properties: asymmetry, inflexibility, transitivity, substitutability and connectedness. Preliminary, this study tested an experimental strategy as a first step toward an investigation on the inclusion of reinforcing stimuli as members of sequential classes. Its aim was to investigate the effect of specific conditioned reinforcers in the formation of ordinal classes. In the presence of one conditional stimulus, six university students had to make sequences of abstract pictures in a determined order that were followed by the presentation of one musical passage. In the presence of another conditional stimulus, the same stimuli had to be sequenced in the reverse order, producing another musical excerpt. Tests showed that in addition to the emergence of defining properties of ordinal classes, the musical excerpts exchanged their reinforcing function for the conditional stimulus function.

Keywords: Ordinal classes, specific conditional reinforcers, humans.

\section{Efecto de los Refuerzos Condicionados Específicos en Clases Ordinales en Humanos}

\section{Resumen}

La explicación según la cual clases de equivalencia son formadas por las contingencias de refuerzo, tiene como implicación que respuestas y estímulos reforzadores también serían incluídos entre sus membros. Soporte empírico para esa tesis viene de experimentos utilizando reforzadores específicos para cada clase potencial, tanto en contexto de discriminación condicional, cuánto de discriminación simple, en este último caso en combinación con reversones sistemáticas, en que las funciones S+e S- de los estímulos discriminativos son constantemente invertidas. En ambos los casos, testes apropiados evidenciaron la inclusión de los estímulos reforzadores como miembros de las clases de equivalencia. Éses resultados permiten suponer que estímulos reforzadores también participarían de clases secuenciales. Éstas son definidas por las propiedades: irreflexividad, asimetría, transitividad, conectividad y substitubilidad. De carácter preliminar, el presente estudio, examinó una estrategia experimental como uno primero paso para una investigación sobre la inclusión de estímulos reforzadores como miembros de clases secuenciales. Su objetivo fue investigar el efecto de reforzadores condicionados específicos en la formación de clases ordinales. En la presencia de uno estímulo condicional, seis universitarios debían secuenciar figuras abstractas en una determinada orden, lo que seguia-se la apresentación de una pasaje musical. En la presencia de otro estímulo condicional, los mismos estímulos debían ser secuenciados, en la orden inversa, producindo otra pasaje musical. Testes evidenciaron que más adelante da emergencia de las propiedades definidoras de clases secuenciales, los pasajes musicales intercambiaron su función reforzadora para la de estímulos condicionales.

Palabras clave: Clases ordinales, reforzadores condicionados específicos, humanos.

Os conceitos de equivalência funcional e de equivalência de estímulos (ou relações de equivalência) são amplamente conhecidos, havendo abundante literatura sobre esses temas, na qual se encontram sobejas apresentações da definição de cada um, de modo que isso se faz dispensável aqui. O leitor interessado poderá encontrar informações detalhadas em vários títulos, dos quais sugerimos: De Rose (1993), Galvão (1993), Sidman (1994) e Sidman e Tailby (1982). 
Numa discussão sobre o que poderia explicar as classes de equivalência, Sidman (2000) propôs que elas são formadas por contingências de reforço, implicando que relações de equivalência consistem de ". . . pares ordenados de todos os elementos positivos que participam na contingência" (p. 128) ${ }^{2}$. Isso significa que as próprias respostas e estímulos reforçadores estão incluídos, além dos estímulos antecedentes. A implicação de que estímulos reforçadores também constituem-se como membros de classes de equivalência tem recebido suporte empírico oriundo de experimentos empregando tanto procedimentos de discriminação condicional (por exemplo, Dube, McIlvane, Mackay, \& Stoddard, 1987) como de discriminação simples (por exemplo, Barros, Lionello-DeNolf, Dube, \& Mcllvane, 2006). A inclusão de respostas como membros de classes de equivalência não será abordada aqui. Para uma introdução à discussão sobre esse tema e as dificuldades inerentes que ele oferece à pesquisa, ver Barros et al. (2006).

\section{Suporte Empírico à Tese de Sidman (2000): Discriminação Condicional}

Dube et al. (1987) relatam três experimentos sobre a inclusão de estímulos reforçadores como membros de classes de equivalência. No primeiro deles foram usados dois conjuntos de estímulos com cada um de dois participantes, um rapaz e uma moça, ambos com atraso no desenvolvimento. Os conjuntos de estímulos eram compostos por nomes falados (N1 e N2), objetos (O1 e O2) e símbolos impressos (S1 e S2). O diferencial inovador nessa pesquisa foi que, em vez de usarem sempre o mesmo reforçador para o ensino de todas as relações, os autores programaram o uso de reforçadores específicos, conforme descrição a seguir.

Para um dos participantes, o procedimento inicial consistiu do ensino das relações N1-S1 e S1-O1, que foram consistentemente reforçadas

2 "These consist of ordered pairs of all positive elements that participate in the contingency" (Sidman, 2000, p. 128). com um determinado item alimentar: F1. Portanto, uma representação mais fiel seria: N1-S1-F1; S1-O1-F1. O ensino das relações entre os estímulos do conjunto 2 seguiu o mesmo padrão: N2-S2-F2; S2-O2-F2. Com o outro participante, o procedimento adotado foi ligeiramente diferente, tendo sido mantido o reforçamento com estímulos reforçadores específicos: N1-O1-F1, O1-S1-F1 e N2-O2-F2, O2-S2-F2. Os itens alimentares usados como reforçadores haviam sido previamente testados por meio de um procedimento que avaliou sua preferência pelos participantes. Eles foram diferentes para o rapaz e para a moça.

Em testes posteriores, sem programação de consequências para as respostas, os participantes apresentaram desempenho que evidenciou a emergência de relações não diretamente ensinadas. Num caso: N1-O1, O1-S1, N2-O2 e O2-S2 e, no outro, N1-S1, S1-O1, N2-S2 e S2-O2, atestando as propriedades de simetria e transitividade que definem as classes como de estímulos equivalentes. A habilidade de emparelhar estímulos por semelhança física, que serve de base para a inferência da reflexividade, também foi demonstrada pelos participantes.

Até aqui, nada de novo. Como dizem os próprios autores: "Demonstrações de unidades comportamentais derivadas após treino de contingências programadas de quatro termos tornaram-se comuns" (Dube et al., 1987, p. 160)․․ A contribuição relevante do estudo veio de um teste no qual os mesmos estímulos que haviam sido usados apenas como reforçadores tiveram agora suas funções alteradas, sendo apresentados como estímulos modelo e/ou de comparação. Nesse novo contexto, os participantes exibiram desempenho consistente com a formação de classes de equivalência que incluíam os estímulos reforçadores como membros: N1-F1, O1-F1, S1-F1, F1-O1, F1-S1 e N2-F2, O2-F2, S2-F2, F2-O2, F2-S2.

3 "Demonstrations of derived behavioral units after training on programmed four-term contingencies have become common place" (Dube et al., 1987, p. 160). 


\section{Suporte Empírico à Tese de Sidman (2000): Discriminação Simples}

Além de estudos utilizando o procedimento mais frequente de discriminação condicional, como o resumido acima, o fenômeno da inclusão de reforçadores como membros de classes de equivalência também foi demonstrado em contexto de discriminação simples. Trabalhando com duas crianças (um menino e uma menina) com nove anos de idade cronológica e idade mental entre 2 e 3 anos, ambas diagnosticadas com autismo, Barros et al. (2006) adotaram um procedimento que alia o uso de reforçadores específicos e a reversão sistemática de discriminações simples.

Resumidamente, pares de estímulos (formas não representacionais) foram apresentados, simultaneamente, um determinado número de vezes consecutivas, em tentativas discretas. Um deles era arbitrariamente definido como $\mathrm{S}^{+} \mathrm{e}$ outro como $\mathrm{S}^{-}$, A escolha de um era reforçada, ao passo que a do outro não. Quando o participante estivesse consistentemente escolhendo o $\mathrm{S}^{+}$, a contingência era revertida, ou seja, a função dos estímulos era invertida, de modo que o antigo $\mathrm{S}^{-}$passava a ser o atual $\mathrm{S}^{+}$e vice-versa. Essa manipulação garante atenção a mudanças nas contingências em vigor e flexibilidade comportamental.

Foram usados dois conjuntos de quatro estímulos cada, que os autores designaram como A1, B1, C1, D1 e A2, B2, C2, D2. Para ilustrar o uso dos reforçadores específicos, digamos que, numa determinada fase do experimento, os pares de estímulos apresentados fossem A1 e A2, sendo o primeiro definido como o $\mathrm{S}^{+}$, isto é, aquele cuja escolha seria seguida de reforço. Os reforçadores, neste caso, foram um estímulo sonoro (S1) e um item alimentar (F1) específicos. Quando a contingência fosse revertida e A2 passasse a ser o $\mathrm{S}+$, então, outro estímulo sonoro (S2) e outro item alimentar (F2), também específicos, seriam usados como reforçadores. Em resumo, a escolha de qualquer um dos estímulos designados pelo numeral 1 , produzia os reforçadores S1 e F1. De modo semelhante, a escolha de qualquer um dos estímulos designados pelo numeral 2, produzia os reforçadores S2 e F2. Desse modo, havia duas classes potenciais de estímulos: a classe 1 e a classe 2 (os itens alimentares foram todos previamente definidos de acordo com a preferência individual dos participantes.)

Sessões de pareamento por identidade usando os mesmos conjuntos de estímulos foram entrepostas às sessões de discriminação simples. Nessa situação, o uso de reforçadores específicos foi mantido, de modo que, por exemplo, dado A1 como estímulo modelo e A1 e A2 como estímulos de comparação, a escolha de A1 seria seguida dos reforçadores S1 e F1. Igualmente, se B2 fosse apresentado como estímulo modelo e B1 e B2 como estímulos de comparação, a escolha de B2 seria reforçada com S2 e F2 e assim por diante. Considerando que o teste crítico para a verificação de formação de classes de equivalência seria em contexto de discriminação condicional, via matching-to-sample (emparelhamento de acordo com o modelo - MTS), sem previsão de reforço para respostas corretas, a introdução gradual de um esquema de reforço intermitente foi usada como providência para preparar os participantes para essa situação.

Os testes de verificação de formação de classes de equivalência foram conduzidos de acordo com o procedimento mais usual de discriminação condicional. $\mathrm{O}$ aspecto relevante a ser destacado aqui é que os participantes foram expostos a uma situação inédita, em que a relação entre os estímulos era arbitrária e não mais de semelhança física. Os resultados mostraram que um dos participantes apresentou desempenho consistente com a formação de classes de equivalência, tendo alcançado o critério definido pelos pesquisadores em todas as relações testadas: AB, BA, AC, CA, AD e DA. Outro participante alcançou o critério apenas nas relações $\mathrm{AB}$ e BA, vindo a apresentar modificação na preferência por itens alimentares e comportamento violento e perturbativo nas sessões, com deterioração do desempenho.

Um ponto central na discussão dos resultados conduzida pelos autores diz respeito à proposição de Sidman (2000), segundo a qual o que explica a equivalência são as contingências de reforço. Eles argumentam que os dados de sua pesquisa oferecem suporte a ela. Adicionalmen- 
te, eles apontam que, em conjunto com outros estudos, seus resultados confirmam descobertas no sentido de que o ensino por meio de emparelhamento de acordo com o modelo não é necessário para a formação de classes. Uma observação ulterior destacada pelos autores é no sentido de que estudos futuros explorassem a possibilidade de que classes de equivalência fossem geradas a partir de contingências de três termos.

\section{Uma Possibilidade a Ser Testada}

As evidências encontradas na literatura que dão suporte à hipótese de estímulos reforçadores como membros de classes de equivalência ensejam a suposição de que eles também participariam como membros de classes sequenciais. Esta é uma linha de pesquisa da Análise do Comportamento que envolve o estudo de relações ordinais, as quais “... . estão presentes no uso de vocábulos relacionais, como, por exemplo, no vocabulário dêitico ... e nos intraverbais, como a contagem, e na organização de frases e sentenças ..." (Ribeiro, Assis, \& Enumo, 2007) e também em relações matemáticas (Nunes \& Assis, 2006).

Antes de prosseguirmos, será esclarecedora uma breve diferenciação entre conceitos que são empregados para se referir a fenômenos que, apesar de possuírem algumas semelhanças são, de fato, bem diferentes. Sequências comportamentais têm sido estudadas enquanto encadeamento de respostas. Tem-se um encadeamento quando uma resposta produz um reforçador condicionado e este funciona, ao mesmo tempo, também como estímulo discriminativo para uma resposta seguinte e assim por diante (Catania, 1998/1999; Millenson, 1967/1975; Skinner, 1953/2003). Um exemplo simples do cotidiano de um grande número de pessoas é o ato de ligar o microcomputador. Até que o equipamento esteja em condições de ser usado, é necessária a emissão de uma série de respostas encadeadas. Primeiro o usuário aperta um botão para ligar o computador. A consequência desse ato é o acionamento do ventilador interno (cooler), cujo ruído típico será ouvido, além do acendimento de alguma lâmpada no painel frontal. $\mathrm{O}$ monitor de vídeo também deverá ser ligado apertando-se um botão, o que gerará, como consequên- cia, o aparecimento de caracteres na tela. Essas primeiras respostas serão consequenciadas com um prompt na tela para que o usuário entre com sua senha. Este é um sinal de que tudo correu bem até agora e que o processo de inicialização pode continuar - reforço para as respostas antecedentes e sinalização da necessidade de uma nova resposta para completar a cadeia. Digitada a senha e pressionada a tecla Enter, após mais alguns segundos será exibida a tela inicial do programa operacional, consequenciando as últimas respostas e sinalizando a possibilidade de emissão de novas respostas.

Apesar de o exemplo acima exigir uma descrição relativamente longa, ele retrata uma cadeia bem simples. Grande parte de nossas ações é, na verdade, constituída de séries de respostas encadeadas e essa é uma área de estudos que despertou o interesse de vários pesquisadores. Avanços, entretanto, mostraram que algumas relações comportamentais envolvendo sequências de estímulos podem emergir sem que tenham sido reforçadas diretamente. Green, Stromer e Mackay (1993) apontaram que essas sequências emergentes não poderiam ser descritas em termos de cadeias comportamentais como a exemplificada no parágrafo anterior (ver também Lima \& Assis, 2003). Inspirados no paradigma de relações de equivalência, eles estenderam o modelo para o âmbito da formação de classes ordinais (ou sequenciais).

À semelhança da definição conceitual de equivalência de estímulos proposta por Sidman e Tailby (1982), Green et al. (1993), baseados em Stevens (1951), também propuseram que classes sequenciais, para serem definidas como tais, devem apresentar um conjunto de propriedades, quais sejam: irreflexividade, assimetria, transitividade e conectividade. A assimetria é uma propriedade cuja característica é a impossibilidade de inversão da ordem de uma relação ordinal aprendida por reforçamento direto; portanto, se um estímulo qualquer, que chamaremos de $\mathrm{A} 1$, for seguido por $(\rightarrow)$ outro A2, então a relação inversa $\mathrm{A} 2 \rightarrow \mathrm{A} 1$ não será verdadeira. Essa é uma propriedade presente em relações do tipo "maior que", "antes que", etc. A irreflexividade_é uma propriedade que determina que um elemento 
não pode ser seguido por ele mesmo: $\mathrm{A} 1 \rightarrow \mathrm{A} 1$ é impossível, pois, uma coisa não é maior que ela mesma, por exemplo. A transitividade é uma propriedade de acordo com a qual, respeitadas as propriedades anteriores, a relação entre pares de estímulos adjacentes será mantida entre pares não adjacentes. Por exemplo, se A1 $\rightarrow$ A2 (par de estímulos adjacentes) e $\mathrm{A} 2 \rightarrow \mathrm{A} 3$ (outro par de estímulos adjacentes), então, A1 $\rightarrow$ A3 (estímulos não adjacentes). A quarta propriedade é a conectividade. Como o nome sugere, ela diz respeito à conexão entre pares de elementos em todas as prováveis combinações, desde que respeitadas as propriedades anteriores. Assim, sejam explicitamente ensinadas as relações A1 $\rightarrow \mathrm{A} 2 \rightarrow \mathrm{A} 3$, por exemplo, deveriam emergir as relações $\mathrm{A} 1 \rightarrow \mathrm{A} 2, \mathrm{~A} 1 \rightarrow \mathrm{A} 3$ e $\mathrm{A} 2 \rightarrow \mathrm{A} 3$. O ensino de pelo menos duas sequências separadas de estímulos possibilita a realização de um teste adicional, o de substituibilidade entre os estímulos. Assim, sejam ensinadas A1 $\rightarrow$ A2 $\rightarrow$ A3 e $\mathrm{B} 1 \rightarrow \mathrm{B} 2 \rightarrow \mathrm{B} 3$, deveriam emergir: $\mathrm{A} 1 \rightarrow \mathrm{B} 2$; $\mathrm{A} 2 \rightarrow \mathrm{B} 3 ; \mathrm{B} 1 \rightarrow \mathrm{A} 3$, etc.

\section{Procedimentos para o Ensino de Classes Ordinais}

$\mathrm{Na}$ literatura encontram-se relatos de dois procedimentos básicos usados para o ensino de sequências de estímulos. Um deles consiste de um aumento gradual do número de estímulos que comporão a sequência final, normalmente limitada a cinco membros. Começa-se reforçando a produção de uma sequência de dois estímulos. Posteriormente, esta é ampliada com a inclusão de um terceiro membro, depois de um quarto e assim por diante. Outro procedimento, denominado "justaposição de pares de estímulos" (overlapping two stimulus sequences), consiste do ensino da produção de sequências de dois pares, exclusivamente. Por exemplo: ensina-se A1 $\rightarrow$ $\mathrm{A} 2$, depois $\mathrm{A} 2 \rightarrow \mathrm{A} 3, \mathrm{~A} 3 \rightarrow \mathrm{A} 4 \mathrm{e}$, finalmente, A4 $\rightarrow$ A5. Esse procedimento evita que alguns estímulos sempre ocupem a extremidade inicial ou final da sequência, como ocorre no procedimento descrito anteriormente, o que pode ser uma fonte de vieses. No procedimento de sobreposição de pares, o segundo elemento de uma sequência será o primeiro na sequência seguinte e assim por diante (para descrições mais detalhadas e uma discussão sobre os dois procedimentos, ver Assis \& Sampaio, 2003; Miccione, Assis, \& Costa, 2010; Souza \& Assis, 2005; Souza, Assis, Magalhães, \& Prado, 2008).

Portanto, no procedimento de ensino por sobreposição dois estímulos são apresentados simultaneamente, devendo o participante responder primeiro a um e depois ao outro para produzir reforço. Então, o estímulo que adquirir função de "primeiro" é removido na tentativa seguinte, mantendo-se o estímulo que adquirir a função de "segundo" e inserindo-se um terceiro estímulo, que deverá adquirir esta função, e assim sucessivamente. Em seguida, o primeiro estímulo é removido, sendo inserido um terceiro, e assim sucessivamente. A literatura tem apontado que, apesar de o ensino por encadeamento favorecer uma aprendizagem sem erros, o ensino por sobreposição de estímulos apresenta resultados mais consistentes com a linha de base adotada sobre a produção de relações ordinais (Assis, Corrêa, Souza, \& Prado, 2010; Assis, Magalhães, Monteiro, \& Carmo, 2011; Souza et al., 2008; Verdu, Souza, \& Lopes, 2006). As relações testadas têm sido conduzidas sem reforçamento diferencial e com base nas relações ensinadas, consistindo na verificação da recombinação de estímulos positivamente relacionados em contingências tríplice de reforçamento.

\section{Produção de Sequências Sob Controle Condicional}

A produção de sequências, assim como o comportamento de um modo geral, é sensível ao controle por estímulos condicionais. Há relatos de experimentos bem sucedidos, nos quais se ensinou que na presença de um estímulo condicional, como um retângulo de uma determinada cor, por exemplo, determinados estímulos deveriam ser sequenciados de acordo com uma determinada ordem, ao passo que, na presença de um segundo estímulo condicional, um retângulo de outra cor, os mesmos estímulos deveriam ser sequenciados na ordem inversa (ver Souza et al., 2008; Souza, Magalhães, Assis, \& Goulart, 2010). Testes sem reforço diferencial possibilitaram a observação de padrões de respos- 
tas sob controle condicional permitindo inferir a emergência de classes sequenciais possuindo as respectivas propriedades definidoras descritas acima (ver Assis et al., 2010; Assis et al., 2011; Souza et al., 2008; Verdu et al., 2006).

$\mathrm{O}$ presente estudo tem caráter preliminar e visa sondar a intercambiabilidade entre as funções reforçadora e condicional de um mesmo estímulo, num contexto de produção de sequências. Para investigar essa possibilidade, foi adotado um procedimento combinando o procedimento de sobreposição de pares de estímulos, a tática de reversões sistemáticas das contingências - sendo que estímulos condicionais diferentes sinalizavam a ordem em que os estímulos discriminativos deveriam ser sequenciados $-\mathrm{e}$ o emprego de reforçadores específicos para cada classe potencial.

\section{Método}

\section{Participantes}

Participaram seis estudantes universitários de diferentes cursos, com idades variando de 20 a 25 anos, sendo três deles do sexo masculino e três do feminino (ver Tabela 1). Um termo de consentimento livre e esclarecido foi assinado pelos participantes conforme exigência da Resolução 196/96 do Conselho Nacional de Saúde (Protocolo n ${ }^{\circ}$ 080/09 - Instituto de Ciências da Saúde da Universidade Federal do Pará [ICS/ UFPA]).

Tabela 1

Número de Participantes por Sexo, Idade e Tipo de Curso Universitário

\begin{tabular}{cccc}
\hline Participantes & Sexo & Idade (anos) & Curso (situação) \\
\hline Sam & F & 20 & Design (cursando) \\
Pal & F & 23 & Arquitetura (cursando) \\
Raf & M & 23 & Publicidade (cursando) \\
Car & F & 20 & Direito (cursando) \\
Dav & M & 25 & Engenharia (cursando) \\
Tom & M & 22 & \\
\hline
\end{tabular}

\section{Ambiente Experimental e Equipamento}

A pesquisa foi realizada em uma sala do Laboratório de Psicologia, na Universidade, medindo aproximadamente $15 \mathrm{~m}^{2}$, refrigerada e com relativo isolamento acústico. Para a coleta dos dados foi utilizado um notebook com sistema operacional Windows XP Professional, processador Pentium III (Intel) e monitor de vídeo de 17 '. O equipamento contou com a instalação de uma versão atualizada do software PROLER (versão 6.4), elaborado em linguagem Java a partir de versões anteriores (Assis \& Santos, 2010), programa esse desenvolvido por Márcio Braga dos Santos especialmente para este tipo de pesquisa.

\section{Estímulos Experimentais}

Foram utilizados três conjuntos de cinco estímulos cada. Desenhados em preto sobre fundo branco, eles são formas não representacionais medindo $2 \mathrm{~cm}$ x $2 \mathrm{~cm}$ e encontram-se reproduzidos na Figura 1, onde são identificados por um código alfanumérico, que indica as classes sequenciais a serem potencialmente formadas: A1-A2-A3-A4-A5, B1-B2-B3-B4-B5 e C1-C2C3-C4-C5. Dois retângulos coloridos, um verde e outro vermelho, foram usados com a função de estímulos condicionais. A tela do computador foi horizontalmente divida em duas partes, sendo a inferior designada como área de escolha e a superior como área de construção. A cada 
tentativa, um estímulo condicional era apresentado no centro da parte superior da tela. Com o cursor sobre ele, um clique no mouse produzia a apresentação de um par de estímulos abstratos na área de escolha, cada um ocupando uma de 12 posições possíveis, aleatoriamente definida pelo próprio programa. Com o cursor posicionado so- bre uma das figuras, ao clique no mouse ela se movia para a área de construção, posicionando-se abaixo do retângulo colorido. Um clique sobre a segunda figura também a movia para a área de construção, fazendo-a posicionar-se à direita da figura movida em primeiro lugar. A área de construção comporta até 10 estímulos alinhados lado a lado.

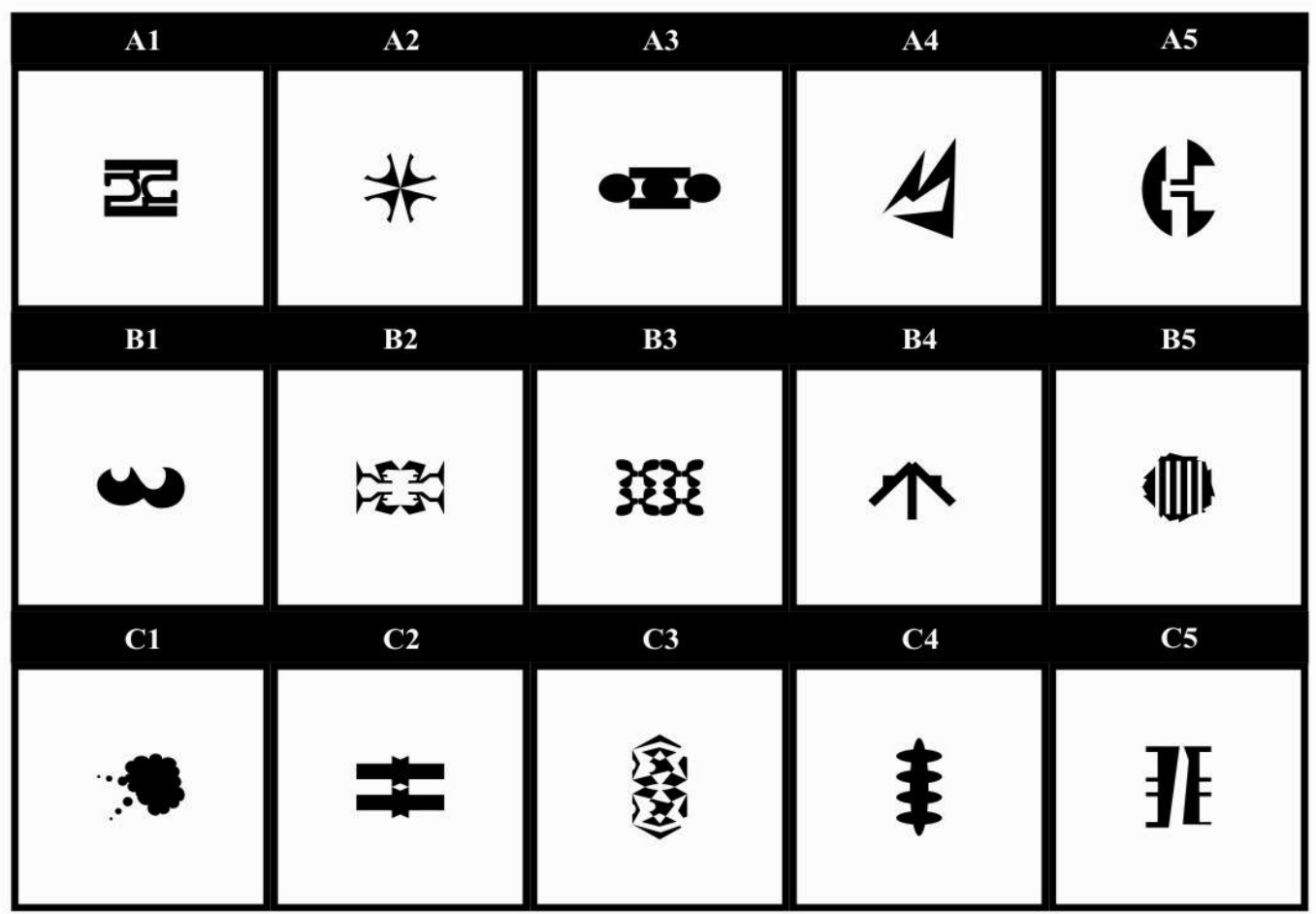

Figura 1. Estímulos experimentais. $O$ código alfanumérico indica as classes sequenciais a serem potencialmente formadas.

\section{Consequências Programadas para as Respostas}

No início da sessão experimental, um levantamento de preferências era conduzido e o experimentador apresentou aos participantes, individualmente, quatro trechos de músicas com duração de aproximadamente quatro segundos. Cada trecho pertence a um de quatro gêneros musicais, a saber: baião, samba, jazz e country. Cada participante escolheu dois trechos, os quais foram, posteriormente, usados como consequências específicas para respostas de produção de sequências definidas como corretas.

Reforço Sonoro 1: Gênero: baião. Duração: 4 segundos.
Reforço Sonoro 2: Gênero: samba. Duração: 4 segundos.

Reforço Sonoro 3: Gênero: jazz. Duração: 4 segundos.

Reforço Sonoro 4: Gênero: country. Duração: 4 segundos.

\section{Procedimento: Linhas Gerais}

O procedimento envolveu dez fases: Ensino de produção de sequências por sobreposição de pares de estímulos, testes de transitividade, teste de substituibilidade, revisão de linha de base, teste de equivalência sequencial 1 com membros das classes $\mathrm{A}$ e $\mathrm{B}$, ensino de produção de sequências e teste de equivalência funcional 
com os membros da classe $\mathrm{C}$, revisão de linha de base com os membros das classes A, B e C, teste de transitividade, teste de substituibilidade e de equivalência sequencial 2 com membros das classes A, B e C.

Os participantes foram recebidos individualmente e levaram cinco sessões experimentais para concluir as tarefas, programadas para durar, no máximo, 25 minutos. Estas ocorreram durante quatro ou cinco dias da semana, sempre no mesmo horário. Se houvesse necessidade, mais de uma sessão poderia ocorrer num mesmo dia, com intervalos de pelo menos 10 minutos entre elas. O participante sentava-se à frente do notebook e o pesquisador permanecia ao seu lado, monitorando a sessão. Ao final de cada sessão, o participante recebia um brinde consistindo de um lanche e uma passagem de ônibus de ida e volta à universidade, independentemente de seu desempenho.

As contingências foram programadas para que a produção de sequências ficasse sob controle de dois diferentes estímulos condicionais, dos quais um deveria controlar a produção da sequência de estímulos numa determinada ordem, ao passo que o outro deveria controlar o sequenciamento dos mesmos estímulos na ordem inversa. Além disso, o procedimento de ensino foi planejado de modo a maximizar as chances de acerto, mantendo reduzida a probabilidade de ocorrência de erros.

Fase 1 - Ensino de Produção de Sequências com Estímulos da Classe A. Nessa fase de ensino de produção de sequências por justaposição de estímulos, ao clicar no mouse estando o cursor sobre o retângulo colorido, dois estímulos (A1 e A2) eram apresentados simultaneamente em duas de doze posições possíveis na área de escolha. O participante era orientado a responder em um dos estímulos de cada vez, recebendo as seguintes instruções: "As figuras irão aparecer aqui [o pesquisador apontava a parte inferior da tela]. Quando aparecerem as figuras, responda em uma e depois na outra". A sequência definida como correta era: A1 $\rightarrow$ A2. Se o participante respondesse corretamente, a consequência seria a apresentação de um dos trechos musicais por ele escolhido. Simultaneamente, o pesquisador fornecia um elogio (por exemplo, "parabéns, você acertou!", "legal”). A sequência descrita foi considerada correta quando produzida na presença do retângulo verde (estímulo condicional), sendo consequenciada especificamente com um dos trechos musicais preferidos do participante. Na presença do retângulo vermelho (outro estímulo condicional), os mesmos estímulos deveriam ser sequenciados na ordem inversa: A2 $\rightarrow$ A1, o que produziria o outro trecho musical escolhido. O participante deveria ser exposto para cada par de estímulos três vezes consecutiva, sem erro, para avançar e ser submetido ao outro par de estímulos (por exemplo, A2 $\rightarrow \mathrm{A} 3$ na presença da cor verde ou A3 $\rightarrow \mathrm{A} 2$ na presença da cor vermelha) e assim por diante, até que todos os pares tivessem sido apresentados na presença dos retângulos coloridos.

\section{Reversão Sistemática da Produção de Sequências}

Considerando que um dos propósitos do estudo era colocar a produção de sequências sob controle de estímulos condicionais, estes eram alternados sistematicamente: após o alcance do critério com um par de estímulos, o estímulo condicional era alterado, invertendo-se a sequência definida como correta. Resumidamente:

$$
\begin{aligned}
& \mathrm{S}^{\mathrm{C}} \mathrm{x}: \mathrm{A} 1 \rightarrow \mathrm{A} 2 \rightarrow \mathrm{S}^{\mathrm{R}} 1 \\
& \mathrm{~S}^{\mathrm{C}} \mathrm{y}: \mathrm{A} 2 \rightarrow \mathrm{A} 1 \rightarrow \mathrm{S}^{\mathrm{R}} 2 \\
& \text { Onde: } \\
& \mathrm{S}^{\mathrm{C}}=\text { Estímulo condicional (retângulo na cor }
\end{aligned}
$$
verde $[\mathrm{x}]$ ou vermelha $[\mathrm{y}]$ ).

$\mathrm{A} 1, \mathrm{~A} 2$ = Estímulos discriminativos a serem sequenciados (conforme Figura 1).

$\rightarrow=$ Seguido por...

$\mathrm{S}^{\mathrm{R}}=$ Trecho de música 1 ou 2 (conforme escolha individual dos participantes).

\section{Procedimento de Correção}

Após responder ao par de estímulos, as respostas consideradas incorretas produziam um escurecimento na tela por quatro segundos, seguidos por uma reapresentação da tentativa 
e uma "dica" do pesquisador, como: "preste atenção", "olhe as duas figuras".

\section{Sondas}

A cada três tentativas consecutivas sem erro, foi intercalada uma sonda, que nada mais era do que uma tentativa idêntica a uma de treino, com a diferença de que não havia consequências programadas para respostas corretas ou incorretas. Nas sondas e em todos os testes, o participante tinha somente uma oportunidade para responder. Caso o participante respondesse diferente do programado pelo experimentador, ele seria reexposto mais uma vez à tentativa anterior de treino com o mesmo par de estímulos.

\section{Ensino de Produção de Sequências com Estímulos da Classe $B$}

Quando sequências com estímulos da classe A estavam sendo consistentemente produzidas sem erros, o mesmo procedimento foi adotado com os estímulos da classe B.

Fase 2 - Testes de Transitividade com Membros das Classes A e B. Após o ensino de produção de sequências, foi aplicado um teste de transitividade com os estímulos da classe A e, posteriormente, com os da classe B. Dois estímulos não adjacentes de uma mesma classe eram expostos na área de escolha e deveriam ser ordenados diretamente na presença da cor verde (por exemplo, $\mathrm{S}^{\mathrm{C}} \mathrm{x}: \mathrm{A} 1 \rightarrow \mathrm{A} 3 ; \mathrm{S}^{\mathrm{C}} \mathrm{x}: \mathrm{B} 2 \rightarrow \mathrm{B} 5$ e assim por diante) e inversamente, na presença da cor vermelha (por exemplo, $\mathrm{S}^{\mathrm{C}} \mathrm{y}: \mathrm{A} 4 \rightarrow \mathrm{A} 2$; $\mathrm{S}^{\mathrm{C}} \mathrm{x}: \mathrm{B} 3 \rightarrow \mathrm{B} 1$ e assim por diante). A ordenação "correta" dos estímulos neste teste evidenciaria a propriedade de transitividade.

Fase 3 - Testes de Substituibilidade entre os Membros das Classes A e B. Após os testes de transitividade, seguiu-se o teste de substituibilidade, cuja finalidade foi a de verificar se o treino anterior havia sido suficiente para que os participantes continuassem respondendo de acordo com as mesmas contingências com pares de estímulos não treinados. Esperava-se que eles os ordenassem de acordo com a mesma ló- gica subjacente ao treino. Isto é, dados estímulos adjacentes de classes diferentes, sua posição na sequência deveria ser mantida. Por exemplo: A1 $\rightarrow \mathrm{B} 2$; A2 $\rightarrow \mathrm{B} 3$, e assim por diante. Além disso, a produção das sequências, se direta ou inversa, deveria ser feita sob controle dos estímulos condicionais. Por exemplo, $\mathrm{S}^{\mathrm{C}} \mathrm{x}: \mathrm{A} 2 \rightarrow \mathrm{B} 3 ; \mathrm{S}^{\mathrm{C}} \mathrm{y}$ : $\mathrm{B} 3 \rightarrow \mathrm{A} 2 \mathrm{e}$ assim por diante.

Fase 4 - Revisão de Linha de Base com Membros das Classes A e B. Após os testes descritos acima, seguiu-se uma revisão da linha de base, cuja finalidade foi a de verificar sua manutenção no repertório de cada participante antes do avanço à fase seguinte. Em termos práticos, a revisão constituiu-se da apresentação de tentativas idênticas às de treino e sondas, alternadamente, com membros das classes A e B.

Fase 5 - Testes de Equivalência Sequencial I com Membros das Classes A e B. Nesta fase experimental, os estímulos usados anteriormente como reforçadores condicionados (trechos de músicas $S^{R} 1$ e $S^{R}$ ), foram agora apresentados na função de estímulos condicionais. Nesta fase, após o participante tocar no quadrado branco (iniciar) que permanecia na parte superior da tela, os estímulos discriminativos eram apresentados em posições aleatórias da área de escolha, simultaneamente a um trecho musical (um dos antigos $S^{R} 1$ ou $S^{R}$ ), o qual durava $4 \mathrm{~s}$. Os estímulos a serem sequenciados foram os das classes A e B. O padrão de respostas previsto foi o de ordenação dos estímulos em ordem direta (por exemplo: A1-A2) na presença de $S^{R} 1$ (agora na função de estímulo condicional) e na ordem inversa (por exemplo: A2-A1) na presença de $\mathrm{S}^{\mathrm{R}} 2$ (agora também na função de estímulo condicional). A confirmação dessa previsão evidenciaria a intercambiabilidade entre as funções reforçadora e condicional dos trechos musicais.

Fase 6 - Treino de Produção de Sequências com Membros da Classe C. Na Fase 6 foi conduzido um treino de produção de sequências com os estímulos da classe C, na presença do $\mathrm{S}^{\mathrm{C}} \mathrm{X}$ (retângulo verde), seguindo-se os mesmos procedimentos da Fase 1. 
Fase 7 - Teste de Equivalência Funcional. Apresentaram-se, em pares justapostos, os estímulos da Classe $\mathrm{C}$ na presença de $\mathrm{S}^{\mathrm{C}} \mathrm{y}$, sem qualquer consequência diferencial programada para a resposta de ordenar inversamente os estímulos (definida como correta). Considerando o princípio de que os efeitos de uma variável aplicada a um estímulo de uma classe estendem-se aos outros membros da mesma classe, previa-se que, em razão do treino anterior com estímulos das classes A e B, o novo treino de produção de sequências com os estímulos da classe $\mathrm{C}$ na presença do $\mathrm{S}^{\mathrm{C}} \mathrm{X}$ fosse suficiente para que os participantes produzissem sequências inversas com esses estímulos quando eles fossem apresentados na presença do $\mathrm{S}^{\mathrm{C}} \mathrm{y}$ (retângulo vermelho), mesmo que isso não tivesse sido diretamente ensinado.

Fase 8 - Revisão da Linha de Base com os Membros das Classes A, B e C. Os participantes foram reexpostos ao treino (e sondas) em relação à produção de sequências com os membros das três classes de estímulos: A, B e C.

Fase 9 - Testes de Transitividade e de Substituibilidade. Foram realizados testes de transitividade entre os estímulos da classe $\mathrm{C}$, por exemplo $\mathrm{S}^{\mathrm{C}} \mathrm{x}: \mathrm{C} 1 \rightarrow \mathrm{C} 4, \mathrm{~S}^{\mathrm{C}} \mathrm{y}: \mathrm{C} 4 \rightarrow \mathrm{C} 1$; e testes de substituibilidade entre os membros das classes A e $\mathrm{C}$ e $\mathrm{B}$ e $\mathrm{C}$, à semelhança do descrito nas Fase 3. Testes de transitividade e de substituibilidade (AC e BC) foram aplicados com todas as combinações possíveis dos membros da Classe $\mathrm{C}$, à semelhança do que foi descrito nas Fases 2 e 3.

Fase 10 - Teste de Equivalência Sequencial II com Membros das Classes A, B e C. O teste final avaliou a inclusão dos estímulos da classe $\mathrm{C}$ como membros das classes de equivalência anteriormente formadas. Os estímulos $\mathrm{S}^{\mathrm{R}} 1$ e $\mathrm{S}^{\mathrm{R}} 2$, que exerceram anteriormente a função de reforçadores condicionados, foram apresentados como estímulos condicionais, semelhante à Fase 5. A previsão era que os participantes produzissem, na presença de $\mathrm{S}^{\mathrm{R}} 1$, as sequências A1-B2-C3, $\mathrm{A} 2-\mathrm{B} 3-\mathrm{C} 4$ e A3-B4-C5; e na presença de $\mathrm{S}^{\mathrm{R}} 2$ as sequências A5-B4-C3, A4-B3-C2 e A3-B2$-\mathrm{C} 1$. Cada uma das sequências era apresentada em tentativas separadas, uma por vez, na área de escolha. Devido a longa exposição às sequências de estímulos na Fase 9 e na atual sem qualquer consequência diferencial, optou-se arbitrariamente por usar três sequências na ordem direta e três na ordem inversa, envolvendo três membros, um membro de cada uma das três classes de estímulos.

\section{Resultados e Discussão}

A Tabela 2 exibe o desempenho dos participantes em todas as fases do experimento, expresso em porcentagens de acertos. De um modo geral, há uma variação de $50 \%$ a $100 \%$, sendo que nas fases de ensino e de revisão de linha de base, todos os participantes apresentaram desempenho máximo. Nos testes, os números sofrem uma queda, a qual é acompanhada de um aumento na variabilidade. Importante recordar que nos testes, havia uma única oportunidade de resposta em cada tentativa. A exposição a sucessivas tentativas de testes poderiam gerar condições para o ensino de novas relações entre estímulos.

Mais especificamente, no teste de transitividade com estímulos do Conjunto A, os acertos vão de $80 \%$ a $100 \%$, ao passo que no mesmo teste com estímulos do Conjunto B, eles ficam entre $70 \%$ e $100 \%$. No teste de substituibilidade entre os estímulos dos conjuntos A e B, a variação fica entre $70 \%$ e $90 \%$. Portanto, o desempenho vai se deteriorando à medida que a complexidade da tarefa aumenta. Considerando os três testes em conjunto, temos que em dois deles um ou dois participantes apresentaram desempenho não muito eloquente, mas, ainda assim, acima do nível do acaso. Se não formos tão rigorosos quanto ao desempenho, o que, de certa forma é compensado com o rigor experimental, conforme recordado no final do parágrafo anterior, podemos afirmar que os dados permitem inferir as propriedades de transitividade e substituibilidade. Por sua vez, isso permite afirmar que o procedimento de ensino foi capaz de gerar classes de estímulos sequenciais.

Um dos testes críticos no experimento foi o de equivalência sequencial I, pois ele avaliou a intercambiabilidade entre as funções reforça- 
Tabela 2

Desempenho dos Participantes, em Porcentagem de Acertos (\%), em Cada Uma das Fases do Experimento

\begin{tabular}{|c|c|c|c|c|c|c|}
\hline \multirow[b]{2}{*}{ Fases do experimento } & \multicolumn{6}{|c|}{ Participantes } \\
\hline & Sam & Pal & Raf & Car & Dav & Tom \\
\hline Ensino de produção de sequências (Conjunto A) & 100 & 100 & 100 & 100 & 100 & 100 \\
\hline Ensino de produção de sequências (Conjunto B) & 100 & 100 & 100 & 100 & 100 & 100 \\
\hline Teste de transitividade (Conjunto A) & 90 & 80 & 90 & 90 & 90 & 100 \\
\hline Teste de transitividade (Conjunto B) & 80 & 90 & 70 & 90 & 100 & 100 \\
\hline Teste de substituibilidade (Conjuntos A e B) & 80 & 70 & 70 & 80 & 90 & 80 \\
\hline Revisão da linha de base (Conjuntos A e B) & 100 & 100 & 100 & 100 & 100 & 100 \\
\hline Teste de equivalência sequencial I (Conjuntos A e B) & 60 & 70 & 80 & 80 & 90 & 90 \\
\hline Ensino de produção de sequências (Conjunto C) & 100 & 100 & 100 & 100 & 100 & 100 \\
\hline Teste de equivalência funcional (Conjunto C) & 100 & 100 & 90 & 100 & 100 & 100 \\
\hline Revisão da linha de base (Conjuntos A, B e C) & 100 & 100 & 100 & 100 & 100 & 100 \\
\hline Teste de transitividade (Conjunto C) & 80 & 100 & 70 & 80 & 90 & 100 \\
\hline Teste de substituibilidade (Conjuntos A e C) & 70 & 80 & 70 & 70 & 80 & 90 \\
\hline Teste de substituibilidade (Conjuntos B e C) & 70 & 80 & 70 & 80 & 80 & 80 \\
\hline Teste de equivalência Sequencial II (Conjuntos A, B e C) & 60 & 60 & 50 & 80 & 80 & 90 \\
\hline
\end{tabular}

dora e condicional dos trechos musicais. Dois participantes apresentaram $60 \%$ e $70 \%$ de acertos, respectivamente, ao passo que, entre os outros quatro, os acertos foram da ordem de $80 \%$ a $90 \%$. Considerando o ineditismo da situação do ponto de vista do participante, em que estímulos que até então seguiam suas respostas agora as antecedem, o fato de eles terem respondido de acordo com o previsto na maioria dos casos é bastante animador, sugerindo que a estratégia experimental proposta tem potencial. Uma outra possibilidade de manipulação seria usar somente estímulos visuais.

No que diz respeito ao desempenho dos participantes com os estímulos do conjunto $\mathrm{C}$, os dados também são encorajadores. Lembrando que houve reforço apenas para sua ordenação direta na presença do $\mathrm{S}^{\mathrm{C}} \mathrm{x}$, no teste em presença do $\mathrm{S}^{\mathrm{C}} \mathrm{y}$ os mesmos estímulos foram ordenados inversamente em $90 \%$ a $100 \%$ das tentativas, numa clara demonstração de equivalência funcional entre os estímulos recém treinados do Conjunto $\mathrm{C}$ e os treinados anteriormente (conjuntos A e B).
Examinemos agora, em conjunto, os testes das propriedades de transitividade e substituibilidade com os estímulos dos conjuntos $\mathrm{A}$ a $\mathrm{C}$. À semelhança dos resultados nos outros testes, os números ficaram entre $70 \%$ e $100 \%$, com a diferença de que os escores menores foram mais frequentes, havendo apenas um caso de escore máximo obtido por um dos participantes no teste de transitividade com estímulos do Conjunto C. Novamente, se considerarmos que o rigor experimental pode compensar um nível não tão elevado de rigor com relação ao desempenho, podemos inferir, a partir dos dados, as propriedades definidoras das classes ordinais.

Finalmente, o segundo teste crítico foi o de equivalência sequencial II, que avaliou a tanto a inclusão dos estímulos $\mathrm{C}$ às classes previamente formadas, como sua relação com os antigos estímulos reforçadores, agora na função de estímulos condicionais. Este foi o teste em que os participantes apresentaram seu pior desempenho: três deles acertaram de $50 \%$ a $60 \%$ das tentativas e outros três de $80 \%$ a $90 \%$. O aumento 
no número de classes, no número de estímulos individuais e, consequentemente, no número de relações entre estímulos, parece ser um fator determinante dessa queda observada, a medida que a complexidade da tarefa aumenta. Todavia, os resultados dos testes de equivalência sequencial I permitem manter a confiança na estratégia experimental, pois metade dos participantes apresentaram resultados consistentes com a linha de base adotada.

Os dados sugerem que a estratégia experimental adotada no experimento aqui relatado pode ser promissora para a integração de estímulos reforçadores a classes de equivalência. Trechos musicais foram usados como reforçadores condicionados. Estímulos desse tipo são facilmente manipuláveis para serem apresentados também como estímulo condicional e os resultados do estudo permitem afirmar, com alguma segurança, que, mesmo sem qualquer treino explícito específico, os estímulos usados inicialmente apenas para consequenciar respostas, também foram eficazes para controlar a ordem em que os estímulos discriminativos seriam sequenciados. Não foram conduzidos testes específicos para verificar a integração dos estímulos reforçadores às classes sequenciais nem a inclusão de todos os estímulos em classes de equivalência.

No caso de verificação de inclusão de reforçadores em classes de estímulos definidas pela função ordinal avalia-se que, neste estudo, seria indicado o uso de estímulos visuais como reforçadores, uma vez que a literatura de formação de classes normalmente aponta o uso de estímulos da mesma propriedade e, posteriormente, para a verificação da inclusão dos estímulos em classes de equivalência através do procedimento de matching-to-sample, os estímulos usados inicialmente como reforçadores iriam figurar como estímulos de comparação, também visuais. Aprimoramentos na técnica experimental serão necessários para que isso seja feito em estudos futuros, por exemplo, usar filme ou animação gráfica.

À luz de estudos sobre a interação entre classes sequenciais e de equivalência (Assis et al., 2011; Nunes \& Assis, 2006; Souza et al., 2008), discutir possibilidade de, em estudos como os relatados, os testes de inclusão de refor- çadores em classes de equivalência serem feitos em contexto de MTS.

Uma das razões pela qual se optou pelo uso de trechos musicais como potenciais reforçadores condicionados foi a facilidade técnica que esses estímulos oferecem para ter intercambiada sua função para a de estímulos condicionais. Os dados sugerem que eles foram eficazes como reforçadores. Contudo, um estudo de Dube, Callahan e McIlvane (1993) mostrou que para os seus sujeitos, que eram ratos, trechos musicais funcionaram melhor como estímulos discriminativos do que como reforçadores. Em que pesem as diferenças entre as espécies em cada caso, para humanos a repetição de um mesmo trecho musical pode levar à saciação rapidamente. Face a essas considerações, sugerimos que, em estudos futuros, opte-se por reforçadores mais potentes.

\section{Referências}

Assis, G., J. A., Corrêa, D. R., Souza, C. B. A., \& Prado, P. S. T. (2010). Aprendizagem de relações ordinais por meio de treino de uma única sequência de estímulos. Psicologia: Teoria $e$ Pesquisa, 26, 165-176. doi:10.1590/S010237722010000400011

Assis, G. J. A., Magalhães, P. G S., Monteiro, P. D. S., \& Carmo, J. S. (2011). Efeitos da ordem de ensino e da transferência de funções sobre relações ordinais em surdos. Acta Comportamentalia, 19, 43-63.

Assis, G. J. A., \& Sampaio, M. E. C. (2003). Efeitos de dois procedimentos de ensino na formação de classes sequenciais. Interação em Psicologia, 7, 53-62.

Assis, G. J. A., \& Santos, M. B. (2010). PROLER (versão 6.4) [Sistema computadorizado de ensino de comportamentos conceituais]. Belém, PA: Universidade Federal do Pará.

Barros, R. S., Lionello-DeNolf, K., Dube, W. V., \& McIlvane, W. J. (2006). Equivalence class formation via identity matching-to-sample and simple discrimination with class specific consequences. Revista Brasileira de Análise do Comportamento, 2, 79-92.

Catania, A. C. (1999). Aprendizagem: Comportamento, linguagem e cognição (D. de Souza, Trad.). Porto Alegre, RS: Artmed. (Original publicado em 1998). 
De Rose, J. C. (1993). Classes de estímulos: Implicações para uma análise comportamental da cognição. Psicologia: Teoria e Pesquisa, 9, 283-303.

Dube, W. V., Callahan, T. D., \& McIlvane, W. J. (1993). Serial reversals of concurrent auditory discriminations in rats. The Psychological Record, 43, 429-440.

Dube, W. V., McIlvane, W. J., Mackay, H., \& Stoddart, L. (1987). Stimulus class membership established via stimulus-reinforcer relations. Journal of Experimental Analysis of Behavior, 47, 159-175. doi:10.1901/jeab.1987.47-159

Galvão, O. F. (1993). Classes funcionais e equivalência de estímulos. Psicologia: Teoria e Pesquisa, 9, 547-554.

Green, G., Stromer, R., \& Mackay, H. (1993). Relational learning in stimulus sequences. The Psychological Record, 43, 599-616.

Lima, M. P. L., \& Assis, G. J. A. (2003). Emergência de classes sequenciais após treino com pareamento consistente. Psicologia: Teoria e Pesquisa, 19, 75-84. doi:10.1590/S010237722003000100010

Nunes, A. L. M., \& Assis, G. J. A. (2006). Emergência de classes ordinais após o ensino de relações numéricas. Revista Brasileira de Análise do Comportamento, 2, 203-219.

Miccione, M. M, Assis, G. J. A., \& Costa, T. D. (2010). Variáveis de controle sobre o responder ordinal: Revisitando estudos empíricos. Perspectivas em Análise do Comportamento, 1(2), 130-148.

Millenson, J. R. (1975). Princípios de análise do comportamento (A. A. Souza, \& D. Rezende, Trads.). Brasília, DF: Coordenada. (Original publicado em 1967)

Ribeiro, M. P. L., Assis, G. J. A., \& Enumo, S. R. F. (2007). Comportamento matemático: Relações ordinais e inferência transitiva em pré-escolares. Psicologia: Teoria e Pesquisa, 23, 25-32. doi:10.1590/S0102-37722007000100004

Sidman, M. (1994). Equivalence relations and behavior: A research story. Boston, MA: Authors Cooperative.
Sidman, M. (2000). Equivalence relations and the reinforcement contingency. Journal of the Experimental Analysis of Behavior, 74, 127-146. doi:10.1901/jeab.2000.74-127

Sidman, M., \& Tailby, W. (1982). Conditional discrimination versus matching to sample: A expansion of the testing paradigm. Journal of the Experimental Analysis of Behavior, 37, 5-22.

Skinner, B. F. (2003). Ciência e comportamento humano (J. C. Todorov \& R. Azzi, Trads.). São Paulo, SP: Martins Fontes. (Original publicado em 1953)

Souza, J. A. N., \& Assis, G. J. A. (2005). Efeito de dois procedimentos de ensino sobre o comportamento de ordenar. Psicologia em Estudo, 10, 527536. doi:10.1590/S1413-73722005000300021

Souza, R. D. C., Assis, G. J. A., Magalhães, P. G. S., \& Prado, P. S. T. (2008). Efeitos de um procedimento de ensino de produção de sequências por sobreposição sob controle condicional em crianças surdas. Interação em Psicologia, 12, 59-75. doi:10.5380/psi.v12i1.9072

Souza, R. D. C., Magalhães, P. G. S., Assis, G. J. A., \& Goulart, P. R. K. (2010). Emergência de relações ordinais sob controle contextual em surdos. Revista Brasileira de Análise do Comportamento, $6(1)$.

Stevens, J. (1951). Mathematics, measurement, and psychophysics. In S. Stevens (Ed.), Handbook of experimental psychology (pp. 1-49). New York: John Wiley and Sons.

Verdu, A. C. M. A., Souza, D. G., \& Lopes, J., Jr. (2006). Formação de classes ordinais após a aprendizagem de sequências independentes. Estudos de Psicologia (Natal), 11, 87-99. doi:10.1590/S1413-294X2006000100011 